\title{
Initial Alignment of Large Azimuth Misalignment Angle in SINS based on Reduced Multiple Fading Factors Strong Tracking CKF
}

\author{
LU Hang, HAO Shun-yi, PENG Zhi-ying and HUANG Guo-rong \\ College of Aeronautics and Astronautics Engineering, Air Force Engineering University, Shanxi, Xi'an 710038, China
}

\begin{abstract}
Aiming at the problem that Cubature Kalman Filter(CKF) has low accuracy and robustness under the condition of strapdown inertial navigation system(SINS) initial alignment due to model error and external disturbance, Reduced Multiple Strong Tracking Cubature Kalman Filter(RMSTCKF) is proposed, and the algorithm flow and suboptimal solution of multiple fading factor are derived. Multiple fading factor can improve tracking ability under each state according to the degree of uncertainty of different states, having stronger adaptability and robustness. Applying RMSTCKF to large azimuth misalignment angle error function described by Euler platform error angle(EPEA), carrying out the simulation under two different conditions, namely noise mismatch and the base is disturbed, and making contrast between RSTCKF and RCKF, the simulation results show that the filter accuracy and convergence rate of RMSTCK when system noise mismatches with true noise are obviously better than RSTCKF and RCKF, having better practical value in engineering.
\end{abstract}

\section{INTRODUCTION}

Practical working environment of SINS is usually complex and uncertain, the result of SINS coarse alignment is large misalignment, thus making linear error model and KF algorithm inapplicable based on the assumption of small misalignment. Article [1] uses the nonlinear error model and nonlinear filter algorithms described by EPEA to solve the problem, but system will be effected by model error, noise and external disturbance, so nonlinear filter sill have low robustness and tracking capability[2,3]. Article [4] proposes strong tracking filter theory, STF can maintain stronger robustness and tracking capability even when facing model uncertainty, noise and big external disturbance[3,4,5]. Article [3] applies STFUKF to initial alignment, but uncertainty of each state vector is different, so it proves that single fading factor can't track each state vector perfectly. MSTUKF is proposed in article [7], which doesn't need to calculate complex Jacobi matrix. Current theory has proved that the accuracy of UKF and CKF can reach the second-order and the third-order respectively, meanwhile, CKF needn't more sampling points and adjustable parameters, having shorter filter time and higher numerical stability $[8,9,10]$.

This article derives reduced CKF on the basis that the distribution characteristics of cubature points in the third-order spherical radial cubature principle and the measurement equation is linear, combing STF with RCKF framework, introducing 2 multiple fading factor matrix, and proposing RMSTCKF that is appropriate to initial alignment. RMSTCKF only needs one cubature transformation without calculating Jacobi matrix, and multiple fading factor can improve tracking ability correspondingly according to the degree of uncertainty in different states. The SINS nonlinear error function described by EPEA in article [1] is applied in this article, and through the simulations done under the condition that noise mismatch and the base is disturbed, it's effective and practical for RMSTCKF.

\section{MULTIPLE FADING FACTOR STF}

The principle of strong tracking filter(STF) is to introduce an adaptive fading factor $\lambda_{k}$ into predicted covariance matrix $\boldsymbol{P}_{k \mid k-1}$, thus making it real-time in adjusting gain matrix $\boldsymbol{K}_{k}$ online. 2 limitations of STF are:

$$
\begin{cases}\boldsymbol{E}\left[\left(\boldsymbol{x}_{k}-\hat{\boldsymbol{x}}_{k}\right)\left(\boldsymbol{x}_{k}-\hat{\boldsymbol{x}}_{k}\right)^{\mathrm{T}}\right]=\min & (\boldsymbol{a}) \\ \boldsymbol{E}\left[\boldsymbol{\varepsilon}_{k+i} \boldsymbol{\varepsilon}_{k}^{\mathrm{T}}\right]=0, k=1,2, \cdots ; i=1,2, \cdots & (\boldsymbol{b})\end{cases}
$$

Formula (a) is the performance index that makes the filter meet the condition of minimum variance estimation. Formula (b) forces the residual sequence of filter to keep orthogonal moment by moment, conquering the problem that the performance of filer decreases when state changes suddenly or the model is unsure[4,5], thus making STF have stronger robustness and tracking capability. 


\subsection{Flow of RMSTCKF}

Set the initial state and covariance matrix of the system are $\boldsymbol{X}_{0 \mid 0}$ and $\boldsymbol{P}_{0}$, and apply the third-order spherical radial cubature principle to the algorithm. The time update and measurement update based on numerical integration from article [6] are shown as following.

\subsubsection{Flow of RMSTCKF}

(1) calculate cubature points $\boldsymbol{X}_{i, k-1 \mid k-1}(i=1, \cdots 2 n)$

$$
\boldsymbol{X}_{i, k-1 \mid k-1}=\boldsymbol{S}_{k-1 \mid k-1} \boldsymbol{\xi}_{i}+\hat{\boldsymbol{x}}_{k-1 \mid k-1}
$$

Where $\boldsymbol{S}_{k-1 \mid k-1}$ is the Cholesky decomposition of $\boldsymbol{P}_{k-1 \mid k-1}$, $\boldsymbol{P}_{k-1 \mid k-1}=\boldsymbol{S}_{k-1 \mid k-1} \boldsymbol{S}_{k-1 \mid k-1}^{\mathrm{T}} . \boldsymbol{\xi}_{i}=\sqrt{n}[1]_{i}$ denotes set of cubature points, $[1]_{i} \in \mathbf{R}^{n}$ denotes the ith element of completely symmetric point set [1] .

$$
[1]=\left\{\left(\begin{array}{c}
1 \\
0 \\
\vdots \\
0
\end{array}\right),\left(\begin{array}{c}
0 \\
1 \\
\vdots \\
0
\end{array}\right), \cdots,\left(\begin{array}{c}
0 \\
0 \\
\vdots \\
1
\end{array}\right),\left(\begin{array}{c}
-1 \\
0 \\
\vdots \\
0
\end{array}\right),\left(\begin{array}{c}
0 \\
-1 \\
\vdots \\
0
\end{array}\right), \cdots,\left(\begin{array}{c}
0 \\
0 \\
\vdots \\
-1
\end{array}\right)\right\}
$$

(2) calculate cubature points $\boldsymbol{X}_{i, k / k-1}^{*}$ transmitted by state function

$$
\boldsymbol{X}_{i, k / k-1}^{*}=\boldsymbol{f}\left(\boldsymbol{X}_{i, k-1 \mid k-1}\right)
$$

(3) calculate state estimation value at present moment

$$
\hat{\boldsymbol{x}}_{k / k-1}=\sum_{i=1}^{2 n} \omega_{i}\left(\boldsymbol{X}_{i, k / k-1}^{*}\right)
$$

Where $\omega_{i}=1 /(2 n)$ is the weight value of $\boldsymbol{X}_{i, k / k-1}^{*}$.

(4) calculate predicted error covariance matrix $\boldsymbol{P}_{k / k-1}^{l}$ without introducing fading factor

$$
\begin{aligned}
\boldsymbol{P}_{k / k-1}^{l}= & \sum_{i=1}^{2 n} \omega_{i}\left(\boldsymbol{X}_{i, k / k-1}^{*}-\hat{\boldsymbol{x}}_{k / k-1}\right)\left(\boldsymbol{X}_{i, k / k-1}^{*}-\hat{\boldsymbol{x}}_{k / k-1}\right)^{\mathrm{T}} \\
& +\boldsymbol{Q}_{k-1}
\end{aligned}
$$

Mark $\boldsymbol{P}_{k / k-1}^{x}=\sum_{i=1}^{2 n} \omega_{i}\left(\boldsymbol{X}_{i, k / k-1}^{*}-\hat{\boldsymbol{x}}_{k / k-1}\right)\left(\boldsymbol{X}_{i, k / k-1}^{*}-\hat{\boldsymbol{x}}_{k / k-1}\right)^{\mathrm{T}}$.

\subsubsection{Measurement update}

(5) calculate state cubature points $\boldsymbol{X}_{i, k \mid k-1}^{l}$ after update

$$
\boldsymbol{X}_{i, k \mid k-1}^{l}=\boldsymbol{S}_{k \mid k-1}^{l} \boldsymbol{\xi}_{i}+\hat{\boldsymbol{x}}_{k \mid k-1}
$$

Where $\boldsymbol{P}_{k \mid k-1}^{l}=\boldsymbol{S}_{k \mid k-1}^{l}\left(\boldsymbol{S}_{k \mid k-1}^{l}\right)^{\mathrm{T}}$.

During initial alignment process, measurement function of system is usually linear, so it can be used to simplify the measurement update process of filter. Remark $\boldsymbol{A}=\sum_{i=1}^{2 n} \omega_{i}\left(\boldsymbol{X}_{i, k / k-1}^{l}-\hat{\boldsymbol{x}}_{k / k-1}\right)\left(\boldsymbol{X}_{i, k / k-1}^{l}-\hat{\boldsymbol{x}}_{k / k-1}\right)^{\mathrm{T}}$, and prove $\boldsymbol{A}=\boldsymbol{P}_{k / k-1}^{l}$.

From the definition of $\boldsymbol{\xi}_{i}=\sqrt{n}[1]_{i}$ explained in step (1), $\boldsymbol{\xi}_{n} \boldsymbol{\xi}_{n}^{\mathrm{T}}$ can be expressed as: $\boldsymbol{\xi}_{n} \boldsymbol{\xi}_{n}{ }^{\mathrm{T}}=\left[\begin{array}{cccc}0 & 0 & \cdots & 0 \\ 0 & 0 & & 0 \\ \vdots & & \ddots & \vdots \\ 0 & 0 & \cdots & 1\end{array}\right]_{n \times n}$

So $\boldsymbol{\xi}_{1} \xi_{1}^{\mathrm{T}}+\boldsymbol{\xi}_{2} \boldsymbol{\xi}_{2}^{\mathrm{T}}+\cdots+\boldsymbol{\xi}_{n} \boldsymbol{\xi}_{n}^{\mathrm{T}}=\boldsymbol{E}_{n \times n}$, and $\boldsymbol{A}$ can be expressed as:

$$
\begin{aligned}
\boldsymbol{A} & =\sum_{i=1}^{2 n} \omega_{i}\left(\boldsymbol{S}_{k \mid k-1}^{l} \boldsymbol{\xi}_{i}\right)\left(\boldsymbol{S}_{k \mid k-1}^{l} \boldsymbol{\xi}_{i}\right)^{\mathrm{T}} \\
& =\omega_{1} \boldsymbol{S}_{k \mid k-1}^{l} \boldsymbol{\xi}_{1} \boldsymbol{\xi}_{1}^{\mathrm{T}}\left(\boldsymbol{S}_{k \mid k-1}^{l}\right)^{\mathrm{T}}+\omega_{2} \boldsymbol{S}_{k \mid k-1}^{l} \boldsymbol{\xi}_{2} \boldsymbol{\xi}_{2}^{\mathrm{T}}\left(\boldsymbol{S}_{k \mid k-1}^{l}\right)^{\mathrm{T}}+\cdots \\
& +\omega_{2 n} \boldsymbol{S}_{k \mid k-1}^{l} \boldsymbol{\xi}_{2 n} \boldsymbol{\xi}_{2 n}^{\mathrm{T}}\left(\boldsymbol{S}_{k \mid k-1}^{l}\right)^{\mathrm{T}} \\
& =\frac{1}{n}\left[\boldsymbol{S}_{k \mid k-1}^{l} \boldsymbol{\xi}_{1} \xi_{1}^{\mathrm{T}}\left(\boldsymbol{S}_{k \mid k-1}^{l}\right)^{\mathrm{T}}+\boldsymbol{S}_{k \mid k-1}^{l} \boldsymbol{\xi}_{2} \boldsymbol{\xi}_{2}^{\mathrm{T}}\left(\boldsymbol{S}_{k \mid k-1}^{l}\right)^{\mathrm{T}}+\cdots\right. \\
& \left.+\boldsymbol{S}_{k \mid k-1}^{l} \boldsymbol{\xi}_{n} \boldsymbol{\xi}_{n}^{\mathrm{T}}\left(\boldsymbol{S}_{k \mid k-1}^{l}\right)^{\mathrm{T}}\right] \\
& =\frac{1}{n} \boldsymbol{S}_{k \mid k-1}^{l}\left(\boldsymbol{\xi}_{1} \boldsymbol{\xi}_{1}^{\mathrm{T}}+\boldsymbol{\xi}_{2} \boldsymbol{\xi}_{2}^{\mathrm{T}}+\cdots+\boldsymbol{\xi}_{n} \boldsymbol{\xi}_{n}{ }^{\mathrm{T}}\right)\left(\boldsymbol{S}_{k \mid k-1}^{l}\right)^{\mathrm{T}} \\
& =\boldsymbol{S}_{k \mid k-1}^{l} \boldsymbol{E}\left(\boldsymbol{S}_{k \mid k-1}^{l}\right)^{\mathrm{T}}=\boldsymbol{P}_{k \mid k-1}^{l}
\end{aligned}
$$

So $\boldsymbol{A}=\boldsymbol{P}_{k / k-1}^{l}$ is proved.

(6) calculate cubature points $\boldsymbol{Z}_{i, k \mid k-1}^{l}$ transmitted by measurement function

$$
\boldsymbol{Z}_{i, k \mid k-1}^{l}=\boldsymbol{H}_{k} \boldsymbol{X}_{i, k \mid k-1}^{l}
$$

(7) calculate measurement prediction value $\hat{z}_{k \mid k-1}$ at time $k$

$$
\begin{aligned}
\hat{\boldsymbol{z}}_{k \mid k-1} & =\sum_{i=1}^{2 n} \omega_{i} \boldsymbol{Z}_{i, k \mid k-1}^{l}=\boldsymbol{H}_{k} \sum_{i=1}^{2 n} \omega_{i} \boldsymbol{X}_{i, k \mid k-1}^{l} \\
& =\boldsymbol{H}_{k} \sum_{i=1}^{2 n} \omega_{i}\left(\boldsymbol{S}_{k \mid k-1}^{l} \boldsymbol{\xi}_{i}+\hat{\boldsymbol{x}}_{k \mid k-1}\right) \\
& =\boldsymbol{H}_{k} \boldsymbol{S}_{k \mid k-1}^{l} \sum_{i=1}^{2 n} \omega_{i} \boldsymbol{\xi}_{i}+\boldsymbol{H}_{k} \sum_{i=1}^{2 n} \omega_{i} \hat{\boldsymbol{x}}_{k \mid k-1} \\
& =\frac{\boldsymbol{H}_{k} \boldsymbol{S}_{k \mid k-1}^{l}}{2 n}\left(\boldsymbol{E}_{n \times n}+\left(-\boldsymbol{E}_{n \times n}\right)\right)+\boldsymbol{H}_{k} \hat{\boldsymbol{x}}_{k \mid k-1} \\
& =\boldsymbol{H}_{k} \hat{\boldsymbol{x}}_{k \mid k-1}
\end{aligned}
$$

It can be seen from formula (8) that the solution procedure of $\hat{z}_{k \mid k-1}$ makes full use of the distribution characteristics of $\boldsymbol{\xi}_{i}$, without calculating $\boldsymbol{X}_{i, k \mid k-1}^{l}$ in formula (6), and reducing one time cubature transformation.

(8) calculate measurement error covariance matrix $\boldsymbol{P}_{z z, k \mid k-1}$ and predicted cross-covariance matrix $\boldsymbol{P}_{x z, k \mid k-1}$ both introducing fading factor at time $k$

Firstly, $\quad \boldsymbol{P}_{z z, k \mid k-1}$ and $\boldsymbol{P}_{x z, k \mid k-1}$ without introducing fading factor can be expressed as:

$$
\begin{aligned}
& \boldsymbol{P}_{z z, k \mid k-1}=\sum_{i=1}^{2 n} \omega_{i}\left(\boldsymbol{Z}_{i, k \mid k-1}-\hat{\boldsymbol{z}}_{k \mid k-1}\right)\left(\boldsymbol{Z}_{i, k \mid k-1}-\hat{\boldsymbol{z}}_{k \mid k-1}\right)^{\mathrm{T}}+\boldsymbol{R}_{k} \\
& =\sum_{i=1}^{2 n} \omega_{i} \boldsymbol{H}_{k}\left(\boldsymbol{X}_{i, k \mid k-1}^{l}-\hat{\boldsymbol{x}}_{k \mid k-1}\right)\left(\boldsymbol{X}_{i, k \mid k-1}^{l}-\hat{\boldsymbol{x}}_{k \mid k-1}\right)^{\mathrm{T}} \boldsymbol{H}_{k}^{\mathrm{T}}+\boldsymbol{R}_{k}{ }^{(9)} \\
& =\boldsymbol{H}_{k} \boldsymbol{A} \boldsymbol{H}_{k}^{\mathrm{T}}+\boldsymbol{R}_{k}=\boldsymbol{H}_{k} \boldsymbol{P}_{k / k-1}^{l} \boldsymbol{H}_{k}^{\mathrm{T}}+\boldsymbol{R}_{k}
\end{aligned}
$$




$$
\begin{aligned}
& \boldsymbol{P}_{x z, k \mid k-1}^{l}=\sum_{i=1}^{2 n} \omega_{i}\left(\boldsymbol{X}_{i, k \mid k-1}^{l}-\hat{\boldsymbol{x}}_{k / k-1}\right)\left(\boldsymbol{Z}_{i, k \mid k-1}-\hat{\boldsymbol{z}}_{k \mid k-1}\right)^{\mathrm{T}} \\
& =\sum_{i=1}^{2 n} \omega_{i}\left(\boldsymbol{X}_{i, k \mid k-1}^{l}-\hat{\boldsymbol{x}}_{k / k-1}\right)\left(\boldsymbol{H}_{k} \boldsymbol{X}_{i, k \mid k-1}^{l}-\boldsymbol{H}_{k} \hat{\boldsymbol{x}}_{k \mid k-1}\right)^{\mathrm{T}} \\
& =\left[\sum_{i=1}^{2 n} \omega_{i}\left(\boldsymbol{X}_{i, k \mid k-1}^{l}-\hat{\boldsymbol{x}}_{k \mid k-1}\right)\left(\boldsymbol{X}_{i, k \mid k-1}^{l}-\hat{\boldsymbol{x}}_{k \mid k-1}\right)^{\mathrm{T}}\right] \boldsymbol{H}_{k}^{\mathrm{T}} \\
& =\boldsymbol{A} \boldsymbol{H}_{k}^{\mathrm{T}}=\boldsymbol{P}_{k \mid k-1}^{l} \boldsymbol{H}_{k}^{\mathrm{T}}
\end{aligned}
$$

And use 2 multiple fading factor matrix introduced in $\boldsymbol{P}_{k / k-1}^{l}$ to replace single fading factor, the form is:

$$
\boldsymbol{P}_{k / k-1}=\lambda_{k} \boldsymbol{P}_{k / k-1}^{x} \lambda_{k}^{\prime}+\boldsymbol{Q}_{k-1}
$$

So $\boldsymbol{P}_{z z, k \mid k-1}$ and $\boldsymbol{P}_{x z, k \mid k-1}$ that introduce fading factor can be expressed as:

$$
\begin{gathered}
\boldsymbol{P}_{z z, k \mid k-1}=\boldsymbol{H}_{k} \boldsymbol{P}_{k / k-1} \boldsymbol{H}_{k}^{\mathrm{T}}+\boldsymbol{R}_{k} \\
\boldsymbol{P}_{x z, k \mid k-1}=\boldsymbol{P}_{k / k-1} \boldsymbol{H}_{k}^{\mathrm{T}}
\end{gathered}
$$

(9) calculate filter gain matrix $\boldsymbol{K}_{k}$

$$
\hat{\boldsymbol{x}}_{k \mid k}=\hat{\boldsymbol{x}}_{k \mid k-1}+\boldsymbol{K}_{k}\left(z_{k}-\hat{\boldsymbol{z}}_{k \mid k-1}\right)
$$

(10) filter state value at current moment

$$
\begin{aligned}
& \hat{\boldsymbol{x}}_{k \mid k}=\hat{\boldsymbol{x}}_{k \mid k-1}+\boldsymbol{K}_{k}\left(z_{k}-\hat{\boldsymbol{z}}_{k \mid k-1}\right) \\
& \boldsymbol{P}_{k \mid k}=\boldsymbol{P}_{k \mid k-1}-\boldsymbol{K}_{k} \boldsymbol{P}_{z z, k \mid k-1}\left(\boldsymbol{K}_{k}\right)^{\mathrm{T}}
\end{aligned}
$$

From the algorithm flow above, it can be seen that the proposed RMSTCKF in this article can get the solution of $\boldsymbol{P}_{k / k-1}, \boldsymbol{P}_{z z, k \mid k-1}$ and $\boldsymbol{P}_{x z, k \mid k-1}$ only by finishing one time cubature transformation, thus making the calculating quantity reduced and making the result satisfy the limitations in formula (1), completing the whole STF afterwards.

\subsection{Sub-optimal solution of multiple fading factor}

The sub-optimal solution of multiple fading factor is[2]:

$$
\begin{aligned}
& \lambda_{k}=\operatorname{diag}\left[\begin{array}{llll}
\lambda_{k}^{1} & \lambda_{k}^{2} & \cdots & \lambda_{k}^{n}
\end{array}\right] \\
& \lambda_{k}^{i}=\max \left(1, \frac{\alpha_{i} \operatorname{tr}\left[\boldsymbol{N}_{k}\right]}{\sum_{i=1}^{n} \alpha_{i} \boldsymbol{M}_{k}^{i i}}\right) \\
& \boldsymbol{N}_{k}=\boldsymbol{V}_{k}-\boldsymbol{H}_{k} \boldsymbol{Q}_{k-1} \boldsymbol{H}_{k}^{\mathrm{T}}-\boldsymbol{R}_{k} \\
& \boldsymbol{M}_{k}=\boldsymbol{F}_{k \mid k-1} \boldsymbol{P}_{k-1 \mid k-1} \boldsymbol{F}_{k \mid k-1}^{\mathrm{T}} \boldsymbol{H}_{k}^{\mathrm{T}} \boldsymbol{H}_{k} \\
& \boldsymbol{e}_{k}=\boldsymbol{z}_{k}-\boldsymbol{H}_{k} \hat{\boldsymbol{x}}_{k \mid k-1} \\
& \boldsymbol{V}_{k}= \begin{cases}\boldsymbol{e}_{1} \boldsymbol{e}_{1}^{\mathrm{T}} & k=0 \\
\frac{\rho \boldsymbol{V}_{k-1}+\boldsymbol{e}_{k} \boldsymbol{e}_{k}^{\mathrm{T}}}{1+\rho} & k>0\end{cases}
\end{aligned}
$$

Where $\boldsymbol{P}_{k / k-1}^{x}$ denotes the state predicted error covariance matrix without considering $\lambda_{k}$ and $\boldsymbol{v}_{k-1}, \boldsymbol{P}_{k / k-1}^{l}$ denotes the state predicted error covariance matrix considering $\lambda_{k}$ and $\boldsymbol{v}_{k-1}, \boldsymbol{P}_{k / k-1}^{l}=\boldsymbol{P}_{k / k-1}^{x}+\boldsymbol{Q}_{k-1} ; \boldsymbol{P}_{k / k-1}^{x z, l}$ denotes cross- covariance matrix without considering $\lambda_{k} ; \boldsymbol{X}_{i, k \mid k-1}^{l}$ denotes cubature points produced by one-step prediction $\hat{\boldsymbol{x}}_{k / k-1}^{l}$ without considering $\lambda_{k} ; \boldsymbol{N}_{k}, \boldsymbol{M}_{k}, \boldsymbol{H}_{k}$ and $\boldsymbol{V}_{0, k}$ are parameter matrix; weakening factor $\beta=3$, forgetting factor $\rho=0.95$.

From article [2] we know that $\boldsymbol{P}_{k / k-1}^{x}$ and $\boldsymbol{H}_{k}$ can be equally expressed as:

$$
\begin{aligned}
\boldsymbol{P}_{k / k-1}^{x} & =\boldsymbol{F}_{k \mid k-1} \boldsymbol{P}_{k-1 \mid k-1} \boldsymbol{F}_{k \mid k-1}^{\mathrm{T}} \\
& =\sum_{i=1}^{m} \omega_{i}\left(\boldsymbol{X}_{i, k / k-1}^{*}-\hat{\boldsymbol{x}}_{k / k-1}\right)\left(\boldsymbol{X}_{i, k / k-1}^{*}-\hat{\boldsymbol{x}}_{k / k-1}\right)^{\mathrm{T}}
\end{aligned}
$$

Because $\boldsymbol{H}_{k}$ is linear measurement matrix, and $\boldsymbol{M}_{k}, \boldsymbol{N}_{k}$ can be expressed as:

$$
\begin{aligned}
& \boldsymbol{M}_{k}=\boldsymbol{P}_{k / k-1}^{x} \boldsymbol{H}_{k}^{\mathrm{T}} \boldsymbol{H}_{k} \\
& \boldsymbol{N}_{k}=\boldsymbol{V}_{k}-\boldsymbol{H}_{k} \boldsymbol{Q}_{k-1} \boldsymbol{H}_{k}^{\mathrm{T}}-\boldsymbol{R}_{k}
\end{aligned}
$$

Put formula (23), (24), (25) into formula (19), (20), the sub-optimal solution of multiple fading factor can be get.

\section{Large azimuth misalignment angle error model}

Set inertial coordinate system is i-frame, earth coordinate system is e-frame, carrier coordinate system is b-frame. Select local geography coordinate system to be navigation coordinate system $\mathrm{n}$-frame, the navigation coordinate system calculated by SINS is p-frame. Due to the influence of different error origin of SINS, the $p$ and $\mathrm{n}$ calculated by navigation computer have misalignment angle error $\phi=\left[\begin{array}{lll}\phi_{x} & \phi_{y} & \phi_{z}\end{array}\right]^{\mathrm{T}}$, which denotes a group of euler angle that rotate from $\mathrm{n}$-frame to $\mathrm{p}$-frame, the rotation order is defined as $\phi_{z}, \phi_{x}, \phi_{y}$. So the coordinate transfer matrix $\boldsymbol{C}_{n}^{p}$ from n-frame to p-frame is:

$$
\begin{aligned}
& \boldsymbol{C}_{n}^{p}=\left[\begin{array}{ccc}
\cos \phi_{y} & 0 & -\sin \phi_{y} \\
0 & 1 & 0 \\
\sin \phi_{y} & 0 & \cos \phi_{y}
\end{array}\right] \cdot\left[\begin{array}{ccc}
1 & 0 & 0 \\
0 & \cos \phi_{x} & \sin \phi_{x} \\
0 & -\sin \phi_{x} & \cos \phi_{x}
\end{array}\right] \\
& \cdot\left[\begin{array}{ccc}
\cos \phi_{z} & \sin \phi_{z} & 0 \\
-\sin \phi_{z} & \cos \phi_{z} & 0 \\
0 & 0 & 1
\end{array}\right] \\
& =\left[\begin{array}{c}
\cos \phi_{y} \cos \phi_{z}+\sin \phi_{y} \sin \phi_{x} \sin \phi_{z} \\
\cos \phi_{x} \sin \phi_{z} \\
\sin \phi_{y} \cos \phi_{z}-\cos \phi_{y} \sin \phi_{x} \sin \phi_{z}
\end{array}\right. \\
& -\cos \phi_{y} \sin \phi_{z}+\sin \phi_{y} \sin \phi_{x} \cos \phi_{z} \quad-\sin \phi_{y} \cos \phi_{x} \\
& \cos \phi_{x} \cos \phi_{z} \quad \sin \phi_{x} \\
& -\sin \phi_{y} \sin \phi_{z}-\cos \phi_{y} \sin \phi_{x} \cos \phi_{z} \quad \cos \phi_{y} \cos \phi_{x}
\end{aligned}
$$

\subsection{SINS attitude error equation}

$\boldsymbol{\omega}_{n p}^{p}$ is the projection of $\mathrm{p}$ to the angular velocity on $\mathrm{n}$, the differential equation of EPEA is[1]: 


$$
\begin{gathered}
\dot{\boldsymbol{\phi}}=\boldsymbol{C}_{\phi}^{-1} \cdot \boldsymbol{\omega}_{n p}^{p} \\
\boldsymbol{C}_{\phi}^{-1}=\frac{1}{\cos \phi_{x}}\left[\begin{array}{ccc}
\cos \phi_{x} \cos \phi_{y} & 0 & \sin \phi_{y} \cos \phi_{x} \\
\sin \phi_{x} \sin \phi_{y} & \cos \phi_{x} & -\sin \phi_{x} \cos \phi_{y} \\
-\sin \phi_{y} & 0 & \cos \phi_{y}
\end{array}\right]
\end{gathered}
$$

From the derivation of $\boldsymbol{\omega}_{n p}^{p}$, it can be expressed as $\boldsymbol{\omega}_{n p}^{p}=\left(\boldsymbol{I}-\boldsymbol{C}_{n}^{p}\right) \boldsymbol{\omega}_{i n}^{n}+\tilde{\boldsymbol{\omega}}_{i n}^{n}-\boldsymbol{C}_{n}^{p} \tilde{\boldsymbol{\omega}}_{i b}^{b}$, then put $\boldsymbol{\omega}_{n p}^{p}$ in formula (27), ignore the second-order and higher-order value, and SINS attitude error function is expressed as:

$$
\dot{\boldsymbol{\phi}}=\boldsymbol{C}_{\phi}^{-1}\left(\left(\boldsymbol{I}-\boldsymbol{C}_{n}^{p}\right) \tilde{\boldsymbol{\omega}}_{i n}^{n}+\boldsymbol{C}_{n}^{p} \delta \boldsymbol{\omega}_{i n}^{n}-\boldsymbol{C}_{b}^{p} \delta \boldsymbol{\omega}_{i b}^{b}\right)
$$

Where $\boldsymbol{C}_{b}^{p}$ is the coordinate transfer matrix from the carrier ( $b$-frame) to computed navigation coordinate( $p$ frame), $\boldsymbol{C}_{n}^{p}$ is the coordinate transfer matrix from the ideal navigation coordinate ( $n$-frame) to computed navigation coordinate ( $p$-frame), $\tilde{\boldsymbol{\omega}}_{i n}^{n}$ denotes the angular rate of the $b$-frame with respect to $n$-frame expressed in $b$-frame, $\delta \omega_{i n}^{n}$ is calculation error of navigation coordinate, $\delta \omega_{i b}^{b}$ is measurement error of gyro.

\subsection{SINS nonlinear filter model}

Assuming that $\delta \omega_{i b}^{b}$ is composed of gyro constant drift $\boldsymbol{\varepsilon}_{b}=\left[\begin{array}{lll}\varepsilon_{b x} & \varepsilon_{b y} & \varepsilon_{b z}\end{array}\right]^{\mathrm{T}}$

and gyro random drift $\boldsymbol{\omega}_{g}=\left[\begin{array}{lll}\omega_{g x} & \omega_{g y} & \omega_{g z}\end{array}\right]^{\mathrm{T}}, \boldsymbol{\omega}_{g}$ is Gaussian white noise with zero mean. $\delta \boldsymbol{f}^{b}$ is composed of accelerometer constant biases $\nabla_{b}=\left[\nabla_{b x} \nabla_{b y} \nabla_{b z}\right]^{\mathrm{T}}$ and accelerometer random drift $\boldsymbol{\omega}_{\boldsymbol{a}}=\left[\begin{array}{lll}\omega_{\mathrm{ax}} & \omega_{\mathrm{ay}} & \omega_{\mathrm{az}}\end{array}\right]^{\mathrm{T}}, \boldsymbol{\omega}_{\boldsymbol{a}}$ is Gaussian white noise with zero mean.

Set the state vector of SINS $\boldsymbol{X}=\left[\begin{array}{llllllllll}\phi_{x} & \phi_{y} & \phi_{z} & \delta v_{x} & \delta v_{y} & \nabla_{\mathrm{b} x} & \nabla_{\mathrm{b} y} & \varepsilon_{\mathrm{b} x} & \varepsilon_{\mathrm{b} y} & \varepsilon_{\mathrm{b} z}\end{array}\right]^{\mathrm{T}}$, specific expression of state matrix $\boldsymbol{f}(\boldsymbol{x})$ can refer to formula (27), (28), (29), (32), state noise vector is $\boldsymbol{w}=\left[\begin{array}{llllllll}w_{\mathrm{gx}} & w_{\mathrm{gy}} w_{\mathrm{gz}} w_{\mathrm{ax}} w_{\mathrm{ay}} & 0 & 0 & 0 & 0 & 0\end{array}\right]^{\mathrm{T}}$. The observation value is velocity error, the observation vector is $\boldsymbol{Z}=\left[\begin{array}{ll}\delta v_{\boldsymbol{x}} & \delta v_{y}\end{array}\right]^{\mathrm{T}}, \quad$ observation matrix is $\boldsymbol{H}=\left[\begin{array}{ll}\boldsymbol{I}_{2 \times 2} & \mathbf{0}_{2 \times 8}\end{array}\right]$, observation noise vector is $\boldsymbol{v}$ that is Gauss white noise with zero mean. So the nonlinear filter model of SINS can be expressed as:

$$
\left\{\begin{array}{l}
\dot{\boldsymbol{X}}=\boldsymbol{f}(\boldsymbol{X})+\boldsymbol{w} \\
\boldsymbol{Z}=\boldsymbol{H} \boldsymbol{X}+\boldsymbol{v}
\end{array}\right.
$$

When the initial alignment is operated with stationary base, the information of longitude, latitude and position is precisely known, and there is no relative motion between $\mathrm{n}$-frame and e-frame, so $\boldsymbol{v}^{n}=0, \boldsymbol{\omega}_{e n}^{n}=0$, $\boldsymbol{\omega}_{i e}^{n}=\left[\begin{array}{lll}0 & \omega_{i e} \cos L & \omega_{i e} \sin L\end{array}\right]^{\mathrm{T}}$, where $L$ denotes geography latitude, $\boldsymbol{f}^{n}=\boldsymbol{C}_{b}^{n} \boldsymbol{f}^{b}=\left[\begin{array}{lll}0 & 0 & -g\end{array}\right]^{\mathrm{T}}$, so the nonlinear initial alignment model of stationary base can be get on the basis of formula (29) and (32).

$$
\left\{\begin{array}{l}
\dot{\boldsymbol{\phi}}=\boldsymbol{C}_{\phi}^{-1}\left(\boldsymbol{I}-\boldsymbol{C}_{n}^{p}\right) \boldsymbol{\omega}_{i e}^{n}-\boldsymbol{C}_{\phi}^{-1} \boldsymbol{C}_{b}^{p} \boldsymbol{\varepsilon}^{b}+\boldsymbol{C}_{\phi}^{-1} \boldsymbol{C}_{b}^{p} \boldsymbol{w}_{g}^{b} \\
\delta \dot{\boldsymbol{v}}^{n}=\left(\boldsymbol{C}_{n}^{p}-\boldsymbol{I}\right) \boldsymbol{C}_{b}^{n} \boldsymbol{f}^{b}+\boldsymbol{C}_{b}^{p} \nabla^{b}+\boldsymbol{C}_{b}^{p} \boldsymbol{w}_{a}^{b} \\
\dot{\boldsymbol{\varepsilon}}^{b}=\boldsymbol{0} \\
\dot{\boldsymbol{V}}^{b}=\boldsymbol{0}
\end{array}\right.
$$

Where the coefficient matrix $\boldsymbol{C}_{\phi}^{-1}$ can be approximated as:

$$
\boldsymbol{C}_{\phi}^{-1}=\left[\begin{array}{ccc}
1 & 0 & \phi_{y} \\
0 & 1 & -\phi_{x} \\
-\phi_{y} & 0 & 1
\end{array}\right]
$$

Where $\sin \phi_{x} \approx \phi_{x}, \cos \phi_{x} \approx 1, \sin \phi_{y} \approx \phi_{y}, \cos \phi_{x} \approx 1$.

\section{Simulation}

Apply RCKF, RSTCKF and RMSTCKF to large azimuth misalignment angle model to carry on I nitial alignment. The position of SINS is $45^{\circ} \mathrm{N}, 108^{\circ} \mathrm{E}$, height equals to $100 \mathrm{~m}$, the initial state of system is $\boldsymbol{X}_{0}=\mathbf{0}$, and the parameters of inertial measurement unit are shown in table 1 .

Table 1. Main parameters of inertial measurement unit

\begin{tabular}{|c|c|}
\hline $\begin{array}{c}\text { Parameters of gyro and } \\
\text { accelerator }\end{array}$ & Value of parameter \\
\hline Gyro constant drift $/\left(\left(^{\circ}\right) / \mathrm{h}\right)$ & 0.1 \\
\hline Gyro random drift $/\left(\left(^{\circ}\right) / \mathrm{h}\right)$ & 0.05 \\
\hline Dynamic range of gyro $/\left(\left(^{\circ}\right) / \mathrm{s}\right)$ & \pm 200 \\
\hline Accelerator constant drift $/ \mu g$ & 100 \\
\hline Accelerator random drift $/ \mu g$ & 50 \\
\hline Dynamic range of accelerator $/ g$ & \pm 30 \\
\hline
\end{tabular}

Set initial value as:

$$
\begin{gathered}
\boldsymbol{P}(0)=\operatorname{diag}\left[\left(2^{\circ}\right)^{2}\left(2^{\circ}\right)^{2}\left(10^{\circ}\right)^{2}(0.1 \mathrm{~m} / \mathrm{s})^{2}(0.1 \mathrm{~m} / \mathrm{s})^{2}\right. \\
\left.(100 u g)^{2}(100 u g)^{2}\left(0.1^{\circ}\right)^{2}\left(0.1^{\circ}\right)^{2}\left(0.1^{\circ}\right)^{2}\right] \\
\boldsymbol{Q}=\operatorname{diag}\left[\left(0.05^{\circ} / h\right)^{2}\left(0.05^{\circ} / h\right)^{2}\left(0.05^{\circ} / h\right)^{2}\right. \\
\left.(50 \mu g)^{2}(50 \mu g)^{2}(50 \mu g)^{2} 0_{5 \times 1}\right] \\
\boldsymbol{R}=\operatorname{diag}\left[(0.1 \mathrm{~m} / \mathrm{s})^{2}(0.1 \mathrm{~m} / \mathrm{s})^{2}\right]
\end{gathered}
$$

\subsection{Initial alignment simulation on stationary base}

Set the initial misalignment angle of E-direction, Ndirection and S-direction is $\phi=\left[\begin{array}{lll}1^{\circ} & 1^{\circ} & 30^{\circ}\end{array}\right]^{\mathrm{T}}$, apply above 3 algorithms to initial alignment simulation for 600 s, the azimuth misalignment angle error is shown in figure 1. From the aspect of convergence, there's little difference between RCKF, RSTCKF and RMSTCKF. Through 100 times Monte-Carlo simulation, the 
accuracy of initial alignment is measured by the RMSE of azimuth misalignment angle at last $100 \mathrm{~s}$, and the specific results are shown in table 2 . From the aspect of convergence speed, RMSTCKF converges around limited accuracy at 120s, faster than RSTCKF and RCKF. System isn't affected by model error and external disturbance in ideal environment, residual sequence keeps orthogonal moment by moment, and the above 3 algorithms can keep better filter capability.

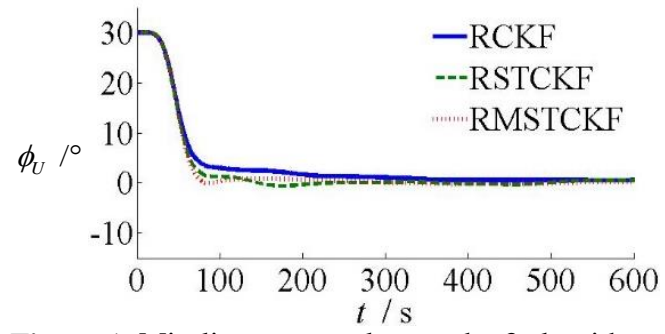

Figure 1. Misalignment angle error by 3 algorithms

Table 2. Alignment results by 3 algrtithms

\begin{tabular}{|c|c|c|c|c|}
\hline $\begin{array}{c}\text { Misalignment } \\
\text { angle error }\end{array}$ & RCKF & RSTCKF & RMSTCKF & $\begin{array}{c}\text { Limited } \\
\text { accuracy(') }\end{array}$ \\
\hline Phi-E & -0.383 & -0.390 & 0.386 & -0.3438 \\
\hline Phi-N & 0.413 & 0.418 & 0.425 & 0.3440 \\
\hline Phi-U & 37.690 & 37.343 & 37.181 & -32.4000 \\
\hline
\end{tabular}

\subsection{Initial alignment simulation under the condition that system noise variance matrix mismatch}

In order to prove the adaptability and robustness of RMSTCKF proposed in this article, expand true noise covariance matrix of system by nine times, the set value of $\boldsymbol{Q}$ in filter model is invariant, and other simulation conditions are the same. The misalignment angle curve in S-direction is shown in figure 2. When system noise mismatches, 3 algorithms all converge, and the filter value at 600 s are respectively 192.36 ', 39.12' and 38.64'. The wave of RCKF is the most obvious, and the wave gradually disappears after 400s, the convergence speed and accuracy of RCKF is bad. Performance of RSTCKF and RMSTCKF are better than RCKF obviously, but there's still small disturbance in RSTCKF, and both of them can converge to limited accuracy quickly.

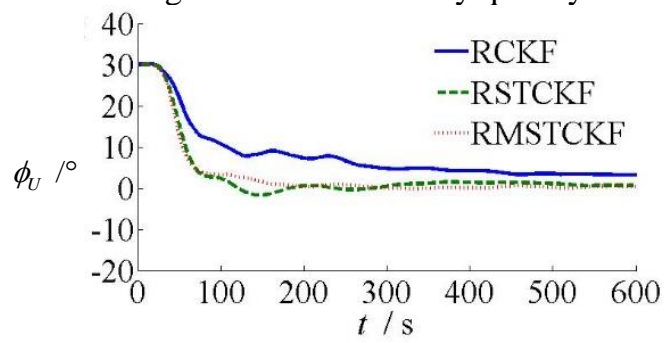

Figure 2. Misalignment angle error of 3 algorithms under the condition that system noise mismatch

\subsection{Initial alignment simulation under the condition that base is disturbed}

Stationary base initial alignment simulation of SINS doesn't consider practical factors of practical working like external disturbance. For the purpose of checking the robustness and tracking ability of RMSTCKF while facing external disturbance, when alignment time reaches $40 \mathrm{~s}$, add sine swaying disturbance which lasts 10 s to the origin of base.

$$
\left\{\begin{array}{l}
\boldsymbol{\theta}=5^{\circ} \sin \left(\frac{2 \pi}{10}(\mathrm{t}-40)\right) \\
\boldsymbol{\gamma}=10^{\circ} \sin \left(\frac{2 \pi}{10}(\mathrm{t}-40)\right)
\end{array} 40<\mathrm{t}<50\right.
$$

Other simulation conditions are invariant, carry on the initial alignment simulation that last 600s with above 3 algorithms. Misalignment angle error curve at Sdirection is shown in figure 3 , figure 3 (a) and figure 3 (b) are enlarged detail where is disturbed.

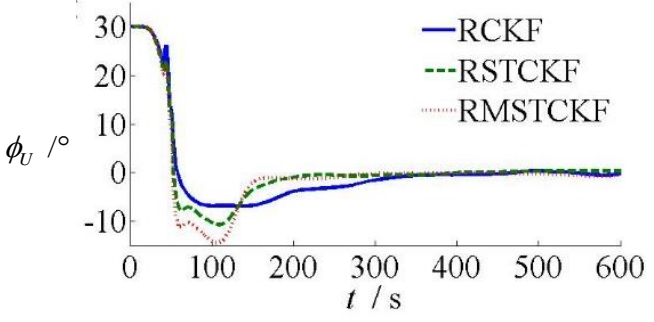

Figure (a). Whole curve of misalignment angle error

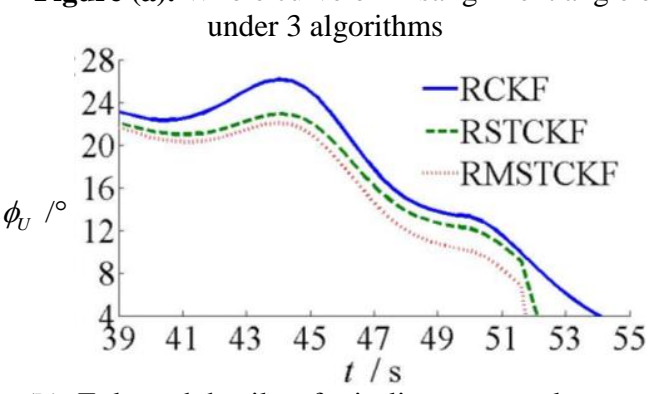

Figure (b). Enlarged detail s of misalignment angle error under 3 algorithms

Figure 3. Misalignment angle error of 3 algorithms while there's sudden disturbance

It can be seen from figure 3 (a) that RMATCKF and RSTCKF have stronger adjustable ability and shorter convergence time than RCKF when system is disturbed. RMSTCKF converges to limited accuracy around 150s, RSTCKF converges to limited accuracy around 220s, and RCKF converges to limited accuracy around 500s.

It can be seen from figure 3 (b) that the filter value of 3 algorithm have different degree of wave due to the disturbance during 40s 50s. However, the disturbance wave of RMSTCKF is obviously smaller than the other 2 algorithms. The disturbance wave of RSTCKF is bigger than RMSTCKF but smaller than RCKF, and the disturbance wave of RCKF is the biggest that filter performance of RCKF is obviously decreasing, being disable to meet the alignment requirements of SINS. From above analysis, it's obvious that the proposed RMSTCKF has better adaptability and higher filter accuracy, equipping with stronger robustness and tracking capability when state changes suddenly.

\section{Conclusion}

An improved algorithm RMSTCKF which is applicable for initial alignment of large azimuth misalignment angle 
is propose in this paper, the calculating flow and suboptimal solution of multiple fading factor matrix are provided. The nonlinear error equation of SINS expressed by EPEA is derived. Complete the initial alignment simulation of large azimuth misalignment angle with 3 algorithms (RCKF, RSTCKF and RMSTCKF) under 3 different conditions that include stationary base, system noise covariance mismatch and the base is disturbed. Results show that RMSTCKF proposed in this paper has stronger adaptability, performing stronger robustness and tracking ability. Compared with the other 2 algorithms, RMSTCKF is better in practical engineering.

\section{References}

1. YAN Gong-min, YAN Wei-Sheng, XU De-min. Application of simplified UKF in SINS initial alignment for large misalignment angles [J]. Journal of Chinese Inertial Technology, 3, 16 (2008)

2. DU Zhan-long, Li Xiao-min. Fault estimation and prediction based on multiple fading factors strong tracking UKF and constrained AR model [J]. Control and Decision, 9, 29 (2014)

3. XUE Hai-jian, Wang Jie, Guo Xiao-song, et al. Design of a Strong Tracking UKF for Nonlinear Self-Alignment of SINS [J]. Journal of Shang Hai
Jiao Tong University, 9, 49 (2015)

4. Zhou D H, FRANK P M. Strong tracking filtering of nonlinear time-varying stochastic systems with colored noise Application to parameter estimation and empirical robustness analysis [J]. International Journal of Control, 2, 65 (1996)

5. Wang L, Wu L, Guan Y, et al. Online sensor fault detection based on an improved strong tracking filter [J]. Sensors, 2, 15 (2015)

6. ZHANG Wen-Jie, Wang Shi-yuan, Feng Ya-li, et al, Huber-based high-degree cubature Kalman tracking algorithm [J]. Acta Phys. Sin., 8, 65 (2016)

7. HU Gao-Ge, Liu Yi-han, Gao Du-Sheng, et al. Improved strong tracking UKF and its application in INS/GPS integr-ated navigation $[\mathrm{J}]$. Journal of Chinese Inertial Technology, 5, 22 (2014)

8. Jia B, Xin M, Cheng Y. High-degree cubature Kalman filter [J]. Automatica, 49 (2013)

9. Pei H L, Sanjeev A, Tharaka A, et al. A Gaussiansum based cubature Kalman filter for bearings only tracking [J]. IEEE Transactions on Aerospace and Electronic Systems, 2, 49 (2013)

10. Lin X G, Xu S S, Xie Y H, Multi-sensor hybrid fusion algorithm based on adaptive square root cubature Kalman filter [J]. Journal of Marine Science and Application, 12 (2013) 